\title{
Triglyceride and high density lipoprotein metabolism in diabetes
}

\begin{abstract}
Atherosclerosis is a major complication of diabetes. The dyslipidaemia of diabetes appears to play an important part in the atherosclerotic process mainly through the disturbance in triglyceride metabolism and the resulting low HDL cholesterol. The article discusses abnormalities in fat metabolism as observed in both chylomicron oin the intestine and very low density lipoprotein (VLDL) in the liver. The impact of disturbed triglyceride metabolism on the atherogenicity is briefly described. Low HDL is a recognised marker of cardiovascular risk. The article describes the relationship between the disturbed triglyceride-rich lipoprotein metabolism found in diabetes and HDL. The effect of diabetes on HDL function is also explored.
\end{abstract}

Keywords: triglycerides; chylomicron; type 2 diabetes; high density lipoprotein; Apolipoprotein A-5; bariatric surgery; Angiopoietin-like protein 3
Volume 5 Issue 5 - 2018

\author{
Gerald H Tomkin \\ University Trinity College Dublin, Ireland
}

Correspondence: Gerald H Tomkin, University Trinity College Dublin, Coillancaraig Lodge, Rocky Valley Drive, Kilmacanogue, Co Wicklow, Ireland, Tel +353 86 8299I54,

Email gerald.tomkin@tcd.ie

Received: July 19, 2018 | Published: September 10, 2018

\section{Introduction}

Atherosclerosis is a major complication of diabetes. Hyperglycaemia, hypertension and dyslipidaemia are the major risk factors found in diabetes. This article reviews the metabolic disturbance which occurs in diabetes that leads to hypertriglyceridaemia and low high density lipoproteins (HDL) and discuss why this profile is atherogenic. Diabetes is a condition in which there is a deficiency of insulin. Absolute deficiency occurs when the beta cells in the pancreas have entirely lost their insulin secreting capacity but most patients with diabetes have some residual function and it is insulin resistance that tips the balance into diabetes when the insulin resistance cannot be overcome by a sufficient increase in insulin production.

\section{Chylomicron and VLDL formation}

Triglycerides are particles consisting of 3 fatty acids attached to glycerol. They are broken down in the intestine into monoacylglycerol and fatty acids, absorbed in the enterocytes and re-esterified primarily through the monoacylglycerol pathway, prior to incorporation into the chylomicron. ${ }^{1-2}$ Another pathway, the glycerol 3 phosphate transfer pathway (GPATH3) has recently been shown to be involved in intestinal lipid metabolism. ${ }^{3}$ The chylomicron is assembled in the intestine from triglyceride, cholesterol, phospholipid and Apolipoprotein (Apo) B 48, a truncated version of Apo B 100, under the influence of microsomal triglyceride transfer protein (MTP) ${ }^{4}$

The chylomicron is taken up from the intestinal wall via the lymphatic system and the triglyceride is transferred to the liver for circulation or stored in adipose tissue. ${ }^{5}$ Fatty acids are released from the liver in the fasting state, converted to triglycerides through 2 steps, diacylglycerol transferase 1 (DGAT 1) to diacylglycerol and DGAT 2 to triglyceride and assembled with the addition of cholesterol and Apo B100, by MTP for transport as very low density lipoprotein (VLDL) or to a lesser extent as low density lipoprotein (LDL). ${ }^{6}$ In the intestine DGAT is predominantly involved with the synthesis of triglyceride and dietary fatty acids. ${ }^{78}$ In the postprandial state esterified lipids generated by intestinal DGAT1 are incorporated into chylomicron particles. Loss of DGAT1, specifically in the intestine, leads to a loss of postprandial triglyceride. ${ }^{9}$ Sachdev et al., ${ }^{10}$ have shown that deficiency/ inhibition of DGAT1 reduces chylomicron size and increases transintestinal cholesterol excretion. Hung et al.,, ${ }^{11,12}$ have shown that DGAT 1 and 2 regulate enterocytes triacylglycerol distribution into distinct subcellular pools.

\section{Central nervous system control of hepatic lipid metabolism}

Type 2 diabetes is associated with fatty infiltration of the liver an inflammatory condition leading to cirrhosis. Neuropeptide Y (NPY) is a neurotransmitter which is involved in many physiological processes. It has been shown to be associated with regulation of food intake. Intracerebroventricular administration of NPY has been shown to activate hepatic regulatory proteins involved in re-modelling phospholipid into triglyceride for VLDL maturation and secretion. They showed that the central nervous system NPY had this effect had this effect through sympathetic signalling to the liver. This has been shown to be due to an increase in endoplasmic reticulum (ER) stress followed by ER autophagy and an increase in fatty acid oxidation. ${ }^{14}$ An investigation into the regulation of hepatic lipid metabolism has shown in an animal model that cathepsin B regulates VLDL secretion and fatty acid uptake via cleavage of liver fatty acid binding protein in the presence of oleic acid. ${ }^{15}$ Inhibition of VLDL secretion reduces plasma levels of Apo B but can result in hepatic steatosis. ${ }^{13}$ Inhibition of Apo B synthesis also reduces VLDL secretion without causing steatosis. As Claud Bernard discovered more than a century ago, the brain through the sympathetic system plays a major part in not only glucose metabolism but also in fat metabolism by the liver.

\section{Cholesterol and triglyceride absorption abnormalities in diabetes}

Serum cholesterol is regulated by Niemann Pick C1-like 1 (NCPl.L-1) which governs absorption. ${ }^{16,17}$ and adenosine triphosphate (ATP) binding cassette proteins (ABC) $\mathrm{G} 5 / 8$ which act as a further gate keeper by excreting excess cholesterol, while fatty acids are almost wholly absorbed in the healthy state. ${ }^{18}$ Thus the chylomicron particle becomes over loaded with triglyceride when fat is eaten in excess leading to moderate increases in triglyceride in the serum. The excess triglyceride is distributed by both increasing the amount of triglyceride in each particle and stimulating more Apo B48 particles thus, not only is the particle triglyceride increased but also the number of particles are increased since there is only one Apo B 48 molecule per particle. MTP assembles the lipoprotein particle by combining 
Apo B48, the solubilising protein, in the intestine and Apo B 100 in the liver with cholesterol, triglyceride and phospholipid. This process is in part regulated by insulin and in diabetes intestinal MTP mRNA is increased with an increase in postprandial Apo B48. ${ }^{19}$ Improvement in blood sugar control reduces post prandial Apo B48. ${ }^{20}$

\section{Chylomicron and atherosclerosis}

Interestingly, inhibition of intestinal MTP reduced fat preference in rats, ${ }^{21}$ and increased HDL-C. ${ }^{22}$ A rare condition causing malabsorption of fatty acids is abetalipoproteinaemia caused by a genetic defect in MTP. This results in fat accumulation in the intestinal mucosa with resulting malabsorption of vitamins and cholesterol, and low serum triglycerides, cholesterol, Apo B48 and Apo B 100. ${ }^{23,24}$

Since the chylomicron particle is mostly a post prandial particle, usually being rapidly cleared by the liver. It is of interest that Irawati et al. ${ }^{25}$ examined in normal subjects the distribution of Apo B48 chylomicron particles after a meal. They divided them up into 3 sizes. The smallest particles with the greatest triglyceride delipidation had a Svedberg flotation rate of $<20$. They found that chylomicronaemia principally consisted of the smallest particles and thus the most atherogenic fraction. It is suggested that the large particles have more difficulty in gaining access to atherosclerotic plaques. However $40 \%$ of subjects demonstrated exaggerated postprandial lipaemia specifically in response to a saturated fatty acid rich meal with a transient shift to more buoyant larger particles. The authors point out that their study suggests the importance in examining the post prandial state when looking at atherogenic triglyceride rich Apo B 48 lipoproteins. In another post prandial study of triglyceride lipolysis in patients with a history of multi factorial chylomicronaemia, the authors found that some patients had a defect in lipolysability and /or hepatic clearance that could be picked up by examining post heparin lipoloysis at 60 min rather than at the more usual 10 minutes, again demonstrating the importance of the post prandial measurements if looking for chylomicron potential atherogenicity. ${ }^{26}$ This on the premise that the smaller particles have greater access to arterial subendothelial space and therefore greater ability to accelerate the atherosclerotic process. ${ }^{27}$

\section{Dyslipidaemia and the beta cell in diabetes}

The relationship between diabetes and alterations in normal lipid metabolism presumes that insulin deficiency/insensitivity is central. More recently it has emerged that altered lipoproteins and in particular LDL may play a role in damaging the beta cell and so promote further beta cell damage in a vicious circle. ${ }^{28}$ LDL receptors are found on the beta cell and an increase in oxidized LDL has been found in diabetes. ${ }^{29,30}$ Masuda $^{31}$ found that after a fat meal, as in the fasted state, the chylomicrons in the blood mostly consisted of the smallest particles and thus the most atherogenic. A recent paper by Natali et al., ${ }^{32}$ investigated whether serum lipids are associated with alterations in insulin secretion or clearance in non diabetic subjects. This was a cross sectional and observational prospective study. Surprisingly LDL cholesterol did not show independent associations with fasting or stimulated insulin secretion or clearance. However, triglycerides showed positive independent associations and HDL cholesterol a negative independent associations with insulin secretion, fasting or after glucose stimulation. The authors conclude that high triglycerides and low HDL cholesterol might contribute to sustain the abnormalities in insulin secretion that characterise the pre diabetic state.

\section{Apolipoprotein A-V diabetes and triglycerides}

Apo $\mathrm{A}-\mathrm{V}$ is involved in the lipoprotein lipase hydrolysis of triglyceride. ${ }^{33}$ There is a strong correlation between single nucleotide polymorphisms in Apo A-V and elevated plasma triglyceride. ${ }^{34}$ Apo A 5 modulates triglyceride levels and has a predictive role in CVD events. ${ }^{35}$ Sharma et al., ${ }^{36}$ have recently reported that in patients with Type 2 diabetes, low levels of Apo A-V are associated with hypertriglyceridaemia and oxidative stress was increased in these patients. Glycation of LDL, is a well known risk factor for CHD as glycated LDL is taken up by the macrophage in an unregulated way and it is more easily oxidised and therefore more atherogenic. ${ }^{37}$ Glycated HDL has been shown to trigger oxidative stress and promote the proliferation and migration of vascular smooth muscle cells, another reason why high HDL in diabetic patients may not be cardioprotective..$^{38}$

Apo A-V is synthesised in the liver and is present in bile. The secretion of Apo A5 is increased by dietary lipid at least in rats. ${ }^{39}$ the authors suggest that the secretion of Apo A-V into the intestinal lumen constitutes a physiological mechanism to regulate the intestinal formation and secretion of chylomicrons.

\section{Weight loss, triglycerides and reversal of diabetes}

The liver $\mathrm{X}$ receptors are hormone receptors that regulate cholesterol absorption through their effect on $\mathrm{ABC}$ G5/8. ${ }^{40,41}$ and in the intestine are involved in the pace of lipid transport. ${ }^{42}$ Since triglyceride in the chylomicron is dependent on diet, it is interesting to examine the changes that occur in starvation. Many studies over the past years have studied short term and long term effects of weight reduction on triglyceride metabolism. ${ }^{43,44}$ Impetus to revive interest has come from effective measures to sustain weight loss in obese patients with and without diabetes through bariatric surgery and more recently through initial low calorie liquid diets and then slow introduction of a more normal diet with supervision. This produced very impressive results at the end of one year in a real world situation as the study was carried out in a variety of general practices. ${ }^{45}$ Remission of diabetes varied with weight loss. Thirty four $\%$ of patients who lost $5-10 \mathrm{~kg}$ and $86 \%$ of those who lost $15 \mathrm{~kg}$ or more reversed their diabetes. Of the 150 patients who commenced the dietary intervention only 32 withdrew demonstrating that in a real world situation, very important benefits can be gained by a structured weight loss program. Serum triglycerides fell by $0.31 \mathrm{mmol} / 1$ - a $20 \%$ drop. ${ }^{46}$ Recently Perry et al have shown in a rat model of Type 2 diabetes, 3 days of semi-starvation leads to reduction of hepatic triglyceride and diacylglycerol content and protein kinase $\mathrm{C}$ (PKC) theta translocation associated with improved insulin sensitivity. ${ }^{47}$ Previously in human studies Taylors group,${ }^{48}$ had shown that in Type 2 diabetes a negative energy diet reduced liver and pancreas triglyceride within 7 days and restored B cell function with normalisation of fasting glucose and even improvement of first phase insulin release. ${ }^{48}$

\section{Life style and dietary intervention}

Lifestyle modification programmes have been shown to reduce cardiovascular risk. Boyer et al., ${ }^{49}$ have shown that at one year after introduction of a lifestyle modification programme in men with abdominal obesity and dyslipidaemia, HDL function and quantity improved.

There has been recognition recently that dietary advice from various national august bodies has been flawed in relation to dietary recommendation and advice about eating eggs and excess cholesterol intake. In the prospective Framingham offspring study Lin et al., ${ }^{50}$ examined dietary cholesterol intake in 993 adults with prevalent impaired glucose tolerance or Type 2 Diabetes. They found that there was no evidence of an adverse association between dietary cholesterol and serum lipid levels or atherosclerotic cardiovascular disease risk. 
This result is perhaps not surprising given the very strong regulation of cholesterol absorption and the feedback mechanisms that upregulate cholesterol synthesis if cholesterol absorption is decreased. A good review of dietary cholesterol and the lack of evidence for cardiovascular disease has recently been published..$^{51}$

\section{Bariatric Surgery}

Bariatric surgery is a well accepted, effective treatment for obesity resulting in very important weight loss and in type 2 diabetes leads to a high remission rate. Adams et al., ${ }^{52}$ reported weight and metabolic outcomes 12 years after gastric bypass. They had 3 groups of patients with severe obesity. Those who sought and underwent Roux-en-Y gastric bypass surgery, a second group were those who sought, but did not undergo, surgery and the third group who did not seek surgery. The patients who had surgery were $45.0 \mathrm{~kg}$ lighter after 12 years. Fifty one percent of these patients with diabetes before the operation had normalised their blood sugars and their diabetes had not reappeared. Triglycerides had fallen from $185 \mathrm{mg} / \mathrm{dl}(2.0 \mathrm{mmol} / \mathrm{l})$ to $103.3 \mathrm{mg} / \mathrm{dl}$ $(1.16 \mathrm{mmol} / \mathrm{l})$ a drop of more than $44 \%$. HDL had risen from 46.6 $\mathrm{mg} / \mathrm{dl}(0.52 \mathrm{mmol} / \mathrm{l})$ to $61.5 \mathrm{mg} / \mathrm{dl}(0.69 \mathrm{mmol} / \mathrm{l})$ a rise of $25 \%$. In the patients who did not have diabetes pre-operation only $3 \%$ developed diabetes at 12 years whereas $26 \%$ of the non-surgery group had diabetes at 12 years. With such a significant drop in triglycerides one would expect a reduction in atherosclerosis and this indeed was found. ${ }^{53,54}$ Ikramuddin et al., ${ }^{55}$ examined lifestyle intervention and medical management with/without Roux-en-Y gastric bypass and hemoglobin A1c (HbAlc), LDL cholesterol, and systolic blood pressure at 5 years in the Diabetes Surgery Study. Adding gastric bypass compared with lifestyle and intensive medical management alone, was significantly better. Other bariatric surgery long term results have also shown a reduction in cardiovascular risk factors and events For example Jakobsen et al. ${ }^{56}$ found after a median of 6.5 years that bariatric surgery as compared to medical treatment resulted in more remission and less new onset hypertension and greater likelihood of diabetes remission at the expense of more depression and treatment with opioids.

\section{Are triglycerides rich particles atherogenic particles.?}

The extent to which effects of body mass index (BMI) on coronary heart disease (CHD) are mediated by glycaemia and lipid risk factors have recently been examined using a two sample Mendelian randomisation method in a very large sample. ${ }^{58}$ The authors conclude that increased triacylglycerol levels and poor glycaemic control appear to mediate much of the effect of BMI on CHD.

Pharmacological reduction in small cholesterol enriched, triglyceride depleted, VLDL has been shown to be associated with a reduction in atherosclerotic cardiovascular disease in the JUPITER trial. ${ }^{59}$ Increased triglycerides have been shown to predict brain betaamyloid and tau pathology 20 years later. ${ }^{60}$

The triglyceride rich particles generate pro-atherogenic LDL and are associated with low HDL, hence hard to tease out the individual risk of the various particles. Treatment to reduce the triglyceride rich particles results in changes to LDL composition and levels as well as increasing HDL. ${ }^{61}$ In the treating to new targets trial (TNT) a post hoc analysis has shown cardiovascular risk was increased in patients who had high triglyceride-rich cholesterol and lowering triglyceride rich cholesterol lipoproteins with atorvastatin yielded benefit over and above lowering LDL cholesterol. ${ }^{62}$

\section{HDL and reverse cholesterol transport}

The examination of large populations investigating the role of genes known to affect serum triglyceride levels have helped to define cardiovascular risk. ${ }^{63}$ Elevated triglyceride-rich lipoproteins represent causal risk factors for low-grade inflammation, atherosclerotic cardiovascular disease, and all-cause mortality. However almost all other studies have failed to distinguish the role of trigly cerides from the inverse relationship with HDL. ${ }^{64-66}$ HDLs are circulating complexes of protein and lipid. The particles vary in size, protein, lipid composition and charge ${ }^{67}$ this explains the many functions attributed to HDL, which include reverse cholesterol transport, inhibition of oxidation, reduction in vascular cell mediated inflammation, and many other functions. ${ }^{68-70}$

Attention is mostly focussed on the ability of HDL to scavenge cholesterol from the periphery through $\mathrm{ABCA} 1$ and deliver it to the liver via the scavenger receptor (SR) B1. Mechanisms are being actively investigated in the hope that HDL therapies may become useful therapies in the treatment/prevention of atherosclerosis. Recently a novel new regulator of ABCA1 expression has been described. ${ }^{71}$ the authors describe trafficking protein kinesin binding 2 (TRAK2) to be a novel regulator of liver X receptor (LXR)-mediated ABCA1 expression, cholesterol efflux and HDL biogenesis.

An alternative pathway has been the uptake of cholesterol by cholesteryl ester transfer protein (CETP) in a two way process, transferring cholesterol from HDL to Apo B-containing lipoproteins while accepting triglyceride from these lipoproteins. The major proteins on HDL are Apo A1 and A2. Apo A1 is synthesised both in the liver and intestine and carried into the circulation on triglyceriderich lipoproteins where it is shed and together with phospholipid forms nascent HDL. ${ }^{72}$ Trigueros-Motos et al. ${ }^{73}$ have shown that ABC A8 facilitates cholesterol efflux and modulates HDL by interacting with Apoliproprotein A1 and potentiates the adenosine triphosphate binding cassette transporter A1-mediated cholesterol efflux.

Through the various roles of HDL, including reverse cholesterol transport, anti-inflammatory, antithrombotic and anti oxidative functions, many studies have shown HDL to be an independent negative risk factor for atherosclerosis. ${ }^{74}$ Apo A1 has been shown in patients with type 2 diabetes to have a stronger effect on preventing cardiovascular disease than HDL. ${ }^{75}$ Borja et al. ${ }^{76}$ have shown that HDL Apo-A1 exchange, a measures of HDL function, is impaired in the metabolic syndrome and this may be a reason why Apo A1 may not be a better marker for CVD risk as compared to HDL-C. The role of Apo $\mathrm{A} 2$ in prevention is less clear, but a recent paper has demonstrated that Apo A2 enhances ABCA1 mediated efflux of cholesterol. The authors suggested that Apo A2 induces a structural change in Apo A1 and this is one mechanism to improve cholesterol acceptance. ${ }^{77}$

Feng et al., ${ }^{78}$ in a cross sectional study of patients with impaired glucose tolerance, found an inverse association between Apo A1 and insulin resistance in these patients. Many studies have shown HDL to be low in type 2 diabetic patients but not type 1. Apo A1 has been independently demonstrated to promote insulin secretion and glucose uptake and to be negatively correlated with insulin resistance. ${ }^{79}$ Understanding the function of HDL requires detailed knowledge of the primary protein Apo A1. This until now has been very difficult however Pourmousa et al., ${ }^{80}$ have recently reported results which they suggest provide a framework for understanding HDL maturation and revise all previous models of nascent HDL. 
Obesity is associated with lower HDL-mediated cholesterol efflux from macrophages and a higher cholesterol ester transfer protein. ${ }^{8}$ Talbot et al., ${ }^{82}$ have surprisingly shown that these parameters of cardiovascular risk are not changed by diet-induced weight loss.

\section{Factors effecting HDL function}

Since HDL contains so many proteins and has such diverse functions it is not surprising that quantity of HDL may not reflect its antiatherogenic function and quality may be more important. This has been demonstrated clearly by the lack of therapeutic benefit with the CETP inhibitors which very successfully rise HDL levels ${ }^{83}$ However the recent results from a trial of Anacetrapib has shown an increase of HDL by $43 \%$ and a reduction in non HDL cholesterol $18 \%$. This was associated with a $1 \%$ difference in primary outcome events specified as first major coronary event or composite of coronary death, myocardial infarction or coronary revascularisation. ${ }^{84}$ the modest effect in benefit may have been due to the reduction in LDL. The manufacturing company Merc Pharmaceuticles have decided not to take the drug forward for licencing

The failure of CEPT inhibitors to show meaningful benefit in cardiovascular prevention terms even though very successful in raising HDL, focused attention on whether it was correct to think of HDL in terms of 'good cholesterol'. Apo A 1 is the major protein associated with HDL and like HDL has also been inversely associated with cardiovascular risk. ${ }^{85}$ Apo A1 has also has also been shown to have anti oxidant anti inflammatory antithrombotic and nitric oxide promoting properties. ${ }^{86,87}$ The association between HDL cholesterol vs Apo A1 with risk of coronary heart disease has been examined in three prospective studies (The Cancer-Norfolk Study, The Atherosclerosis Risk in Communities Study and the Womans Health Study). ${ }^{88}$ The study found that ApoA1 levels do not offer predictive information over and above HDL-C. Surprisingly, in some quartiles of HDL-C, ApoA1 was associated with higher risk of CHD events! Interestingly Sun Y et al. ${ }^{89}$ have shown that older people with diabetes who have higher serum HDL-C had better executive function. The authors followed 152 subjects with normal cognitive function and 119 who had impaired function and re-examined them after 27 months.

There are many reasons why LDL cholesterol in diabetic patients with hyperglycaemia, may be atherogenic, such as increased glycation and increased oxidation. It is less clear that glycation of Apo A1 alters HDL function. Domingo-Espin et al., ${ }^{90}$ have recently shown that glycation of ApoA1 led to loss of lipid binding capability and a reduced ability to catalyse cholesterol efflux from macrophages. Modification of Apo A1 also affected in vivo glucose clearance

Serum amyloid A (SAA) is an inflammatory protein that associates with HDL and causes HDL to become dysfunctional. It has been suggested that solubilisation of membranes by SAA provides a first line of defence in clearing debris from injured sites. ${ }^{91}$ SAA has also been shown to increase vascular smooth muscle cell proliferation. It has been suggested that the contractile vascular smooth muscle phenotype de-differentiates following injury leading to vascular lesions. ${ }^{92}$ Zhang $\mathrm{X}$ et al. ${ }^{93}$ have shown that SAA induced vascular smooth muscle cell phenotype switch, thus potentially promoting atherosclerotic lesions. Griffiths et al., ${ }^{94}$ have shown in young female type 2 diabetic patients an increase in SAA concentrations and dysfunctional HDL features with lower paraoxinase- 1 activity.

Apo C 111, which is an important protein attached to triglyceriderich lipoproteins and inhibits lipoprotein lipase and uptake by the
Apo B receptor, is also found on HDL particles and has been shown to effect HDL mediated cholesterol efflux. ${ }^{95}$ They showed Apo C111 attached to HDL to be a strong predictor of cardiovascular risk whereas HDL without Apo C111 was inversely associated with diabetes cardiovascular risk. ${ }^{96}$

\section{Angiopoietin-like protein 3 and 4}

Angiopoietin-like protein 3 and 4 are glycoproteins involved in angiogenic regulation..$^{97,98}$ It is an important regulator of lipoprotein metabolism. The n-terminal region mediates the inhibition of lipoprotein lipase whereas the C-terminal region is involved in cell adhesion and migration. ${ }^{98}$ There is an inverse correlation between angiopoietin like protein-4 and HDL cholesterol. ${ }^{99,100}$ Plasma levels of angiopoietin-like protein 4 are increased in type 2 diabetes. ${ }^{101}$ Angiopoietin-like protein4 is present in HDL. ${ }^{102}$ Yang et al., ${ }^{103}$ have shown that angiopoietin-like protein 4 on HDL protected HDL from hydrolysis. In type 2 diabetes angiopoietin-like protein 4 levels are increased but the inhibitory effect on endothelial lipoprotein lipase was diminished with a compromised inhibitory effect on endothelial lipase leading to increased HDL hydrolysis and dysfunction. ${ }^{104}$ The authors suggest that improved glycaemic control would result in a lowering of angiopoietin-like protein 4 and improvement in HDL function and therefore reduce cardiovascular risk. Angiopoietinlike protein 3 is an endogenous inhibitor of lipoprotein lipase that is related to angiopoietin-like protein4. Rare loss of function variants are associated with decreased triglyceride levels and lower LDL and HDL. ${ }^{105}$ Deletion of angiopoietin-like protein 3 has been shown to reduce the development of atherosclerosis in Apo E deficient mice. ${ }^{106}$ Dewey et al., ${ }^{107}$ examined the relationship between angiopoietinlike protein 3 loss of function variants and coronary artery disease and found that the loss of function variants had significantly lower triglyceride, HDL cholesterol and LDL cholesterol. Loss of function variants were found in $0.33 \%$ of case variant patients as compared to $0.45 \%$ of controls $\mathrm{P}<0.004)$. In dyslipidaemic mice, inhibition of angiopoietin-like protein 3 resulted in a greater area of atherosclerotic lesion and necrotic lesion area. In another study Graham et al., ${ }^{108}$ using an antisense oligonucleotide targeting angiopoietin 3 mRNA, triglycerides fell by $33-63 \%$, LDL cholesterol by $1.3-34 \%$, VLDL reduced by $28-60 \%$ and Apolipoprotein C111 by $19-59 \%$. In treated mice similar lipid reductions occurred with reductions in liver triglyceride content and progression to atherosclerosis. There was also an improvement in insulin sensitivity. These studies represent a fresh frontier in the treatment of atherosclerosis and coronary artery disease. ${ }^{109}$ Interestingly HDL levels also fell. Angiopoietin-like protein 3 inhibits in vitro endothelial lipase, an enzyme involved in HDL catabolism and has been shown to be positively correlated to plasma HDL cholesterol and phospholipid levels, high triglycerides, low HDL and cardiovascular risk. ${ }^{110}$

\section{High triglycerides, low HDL and diabetes}

The metabolic syndrome is associated with insulin resistance obesity and diabetes. Low HDL and high triglycerides are a common feature in the syndrome and impairment in the functionality of HDL. ${ }^{111}$ A life style weight reduction intervention programme was examined in patients with the metabolic syndrome. ${ }^{112}$ The antioxidant capacity of HDL was significantly improved but only in patients who had normal LDL cholesterol. The improvement in function was associated with improvement in ApoA-1 content and core lipid composition. A surprising result but only 33 patients and only a 12 week study. High triglycerides and low HDL predicted the development of incident 
coronary heart disease and stroke particularly in those with diabetes. ${ }^{113}$ High triglycerides and low HDL were found in a retrospective study of 47000 patients attending Italian diabetes centres to predict the development of diabetic kidney disease. ${ }^{114}$

Pre-beta1 HDL is a major acceptor of free cholesterol from cells. Shiu et al., ${ }^{115}$ have recently shown that pre-beta1 HDL was significantly decreased in Type 2 diabetic patients without cardiovascular disease compared to controls and was associated with decreased cholesterol efflux in cultured cells mediated by ABCA1. The authors suggest that impairment of cholesterol efflux in Type 2 diabetic patients may be a cause for the increased risk of atherosclerosis that these patients carry.

\section{Conclusion}

One way to examine the cardiovascular risk of high triglycerides and low HDL is to examine whether variants in genes that determine the levels of HDL and triglycerides are associated with vascular events. Voigt et al., ${ }^{116}$ examined 14 common single nucleotide polymorphisms (SNP) that exclusively associate with HDL cholesterol and one SNP in the endothelial lipase gene. They found that some genetic mechanisms that raise HDL cholesterol do not seem to lower risk of myocardial infarction.

In a recent excellent review Dron and Hegely. ${ }^{117,118}$ state that definitive evidence for a direct causal role of triglycerides in cardiovascular disease still eludes our grasp. However, there seems to us to be too much evidence already available to suggest definite risk that it would be unwise to ignore the dangers of high triglycerides. Treatment of hypertriglyceridaemia over and above treating LDL to target, in 2018 would seem to be the wise option. HDL and Apo A1 are useful markers of cardiovascular disease risk but treatment to improve function rather than to increase HDL levels seems the new goal of treatment to prevent cardiovascular disease. ${ }^{119}$

\section{Summary}

The article discusses new research into the relationship between the brain and the liver. The role of insulin in lipid metabolism is discussed with particular reference the disturbances that occur in chylomicron metabolism in diabetes. Apo A5 and lipoprotein lipase hydrolysis of triglyceride is discussed. The chylomicron is dependent on dietary intake. New insights from bariatric surgery and starvation diets are presented. The interdependence between triglyceriderich lipoproteins and HDL is explored. HDL function in diabetes is discussed including the role of Apo C111 and the angiopoietin-like lipoproteins attached to HDL. Cardiovascular disease is prominent in diabetes. Triglyceride metablolism is a major disturbance in lipid metabolism generating a cascade of atherogenic particles eventually leading to dysfunctional HDL. There is a great deal of evidence to suggest that triglyceride-rich lipoproteins are atherogenic. The interdependence of these lipoproteins with HDL and LDL have in the past obscured the atherogenic role of atherogenic triglyceride-rich lipoproteins. The lipoprotein cascade starts with dietary cholesterol and triglyceride via the chylomicron. Most of the time now days we are in a postprandial phase, with an increase in grazing. It is likely that lipoprotein patterns have changed over the past number of years and may at least in part be responsible for the increase in diabetes, obesity and atherosclerosis.

\section{Acknowledgments}

None.

\section{Conflict of interests}

The author declares there is no conflict of interest.

\section{Referenece}

1 Kalinowska A, Orlińska B, Panasiuk M, Jamiołkowska M, Zasim A et al. Assessment of preservation of beta-cell function in children with longstanding type 1 Diabetes with "ultrasensitive c-peptide" method. Pediatr Endocrinol Diabetes Metab. 2017;23:130-138.

2 Davis AK, DuBose SN, Haller MJ, et al, T1D Exchange Clinic Network. Prevalence of detectable C-Peptide according to age at diagnosis and duration of type 1 diabetes. Diabetes Care. 2015 ;38:476-481.

3 Khatun I, Clark RW, Vera NB, et al. JTT-130, a novel intestine-specific inhibitor of microsomal triglyceride transfer protein, reduces food preference for fat. J Diabetes Res. 2014;2014:583752.

4 Suzuki T, Swift LL. Discovery of Novel Splice Variants and Regulatory Mechanisms for Microsomal Triglyceride Transfer Protein in Human Tissues. Sci Rep. 2016 ;3;6:27308.

5 Masuda D1, Yamashita S. Postprandial Hyperlipidemia and Remnant Lipoproteins. J Atheroscler Thromb. 2017;24(2):95-109.

6 Okerberg C, Goodwin B Characterization of a Novel Intestinal Glycerol3-phosphate Acyltransferase Pathway and Its Role in Lipid Homeostasis. J Biol Chem. 2016 ;291:2602-2615.

7 Iqbal J, Hussain MM. Intestinal lipid absorption. Am J Physiol Endocrinol Metab. 2009;296:E1183-E1194.

8 Buhman KK, Smith SJ, Stone SJ, et al. DGAT1 is not essential for intestinal triacylglycerol absorption or chylomicron synthesis. J Biol Chem. 2002;277(28):25474-25479

9 Ables GP, Yang KJ, Vogel S, et al. Intestinal DGAT1 deficiency reduces postprandial triglyceride and retinyl ester excursions by inhibiting chylomicron secretion and delaying gastric emptying. J Lipid Res. 2012;53(11):2364-2379.

10 Sachdev V, Leopold C, Bauer R, et al. Novel role of a triglyceridesynthesizing enzyme: DGAT1 at the crossroad between triglyceride and cholesterol metabolism Biochim Biophys. Acta. $2016 ; 1861(9 \mathrm{Pt}$ A):1132-1141.

11 Hung YH, Carreiro AL, Buhman KK. Dgat1 and Dgat2 regulate enterocyte triacylglycerol distribution and alter proteins associated with cytoplasmic lipid droplets in response to dietary fat. Biochim Biophys Acta. 2017;1862(6):600-614.

12 Rojas JM, Bruinstroop E, Printz RL, et al. Central nervous system neuropeptide $Y$ regulates mediators of hepatic phospholipid remodeling and very low-density lipoprotein triglyceride secretion via sympathetic innervation. Mol Metab. 2015;4(3):210-221.

13 Choi SH1, Ginsberg HNIncreased very low density lipoprotein (VLDL) secretion, hepatic steatosis, and insulin resistance. Trends Endocrinol Metab. 2011;22(9):353-363.

14 Conlon DM, Thomas T, Fedotova T, et al. Inhibition of apolipoprotein B synthesis stimulates endoplasmic reticulum autophagy that prevents steatosis. J Clin Invest. 2016;126(10):3852-3867.

15 Thibeaux S, Siddiqi S, Zhelyabovska O, et al Cathepsin B regulates hepatic lipid metabolism by cleaving liver fatty acid-binding protein. $J$ Biol Chem. 2018;293(6):1910-1923.

16 Xie P, Zhu H, Jia L, et al. Genetic demonstration of intestinal NPC1L1 as a major determinant of hepatic cholesterol and blood atherogenic lipoprotein levels. Atherosclerosis. 2014;237(2):609-617.

17 Jia L, Betters JL, Yu L. Niemann-pick C1-like 1 (NPC1L1) protein in intestinal and hepatic cholesterol transport. Annu Rev Physiol. 2011;73:239-259. 
18 Reeskamp LF, Meessen ECE, Groen AK, Transintestinal cholesterol excretion in humans. Curr Opin Lipidol. 2018;29(1):10-17.

19 Phillips C, Mullan K, Owens D, et al. Intestinal microsomal triglyceride transfer protein in type 2 diabetic and non-diabetic subjects: the relationship to triglyceride-rich postprandial lipoprotein composition. Atherosclerosis. 2006;187(1):57-64.

20 Tomkin GH, Owens D. Dyslipidaemia of diabetes and the intestine. World J Diabetes. 2015;6:970-7.

21 Mera Y, Hata T, Ishii Y, et al. JTT-130, a novel intestine-specific inhibitor of microsomal triglyceride transfer protein, reduces food preference for fat. J Diabetes Res. 2014; 2014:583752.

22 Mera Y, Kawai T, Ogawa N, et al. JTT-130, a novel intestine-specific inhibitor of microsomal triglyceride transfer protein, ameliorates lipid metabolism and attenuates atherosclerosis in hyperlipidemic anima models. J Pharmacol Sci. 2015;129(3):169-176.

23 Liu Y, Conlon DM, Bi X, et al. Lack of MTTP Activity in Pluripotent Stem Cell-Derived Hepatocytes and Cardiomyocytes Abolishes apoB Secretion and Increases Cell Stress. Cell Rep. 2017;19 (7):1456-1466.

24 Lee J, Hegele RA .Abetalipoproteinemia and homozygous hypobetalipoproteinemia: a framework for diagnosis and management. J Inherit Metab Dis. 2014;37(3):333-339.

25 Irawati D, Mamo JC, Slivkoff-Clark KM, et al. Dietary fat and physiological determinants of plasma chylomicron remnant homoeostasis in normolipidaemic subjects: insight into atherogenic risk. $\mathrm{Br} J$ Nutr. 2017;117(3):403-412.

26 Marmontel O, Di Filippo M, Marcais C, et al. Alterations in plasma triglycerides lipolysis in patients with history of multifactorial chylomicronemia. Atherosclerosis. 2017;265:22-28.

27 Proctor SD, Vine DF \& Mamo JCL . Arterial permeability and efflux of apolipoprotein B-containing lipoproteins assessed by in situ perfusion and three-dimensional quantitative confocal microscopy. Arterioscler Thromb Vasc Biol. 2004;24:2162-2167

28 Sharma RB, Alonso LC. Lipotoxicity in the pancreatic beta cell: not just survival and function, but proliferation as well?. Curr Diab Rep 2014;14(6):492.

29 Tomkin GH, Owens D. The chylomicron: relationship to atherosclerosis. Int J Vasc Med. 2012;2012:784536.

30 Hasnain SZ, Prins JB, McGuckin MA. Oxidative and endoplasmic reticulum stress in $\beta$-cell dysfunction in diabetes. $J$ Mol Endocrinol. 2016;56(2):33-54

31 Masuda D, Yamashita S. Postprandial Hyperlipidemia and Remnant Lipoproteins. J Atheroscler Thromb. 2017;24(2):95-109.

32 Natali A, Baldi S, Bonnet F, et al. RISC Investigators. Plasma HDLcholesterol and triglycerides, but not LDL-cholesterol, are associated with insulin secretion in non-diabetic subjects. Metabolism. 2017;69:33-42.

33 Merkel M, Loeffler B, Kluger M, et al. Apolipoprotein AV accelerates plasma hydrolysis of triglyceride-rich lipoproteins by interaction with proteoglycan-bound lipoprotein lipase. J Biol Chem. $2005 ; 280(22): 21553-1560$.

34 Sharma V, Forte TM, Ryan RO. Influence of apolipoprotein A-V on the metabolic fate of triacylglycerol. Curr Opin Lipidol. 2013;24:153-159

35 Dallinga-Thie GM, van Tol A, Hattori H, et al. DALI study group Plasma apolipoprotein A5 and triglycerides in type 2 diabetes. Diabetologia. 2006;49(7):1505-1511

36 Sharma D, Garg S, Mehndiratta M, et al .Relationship of Serum Apolipoprotein A-V Levels, Oxidative Stress and Inflammatory Biomarkers with Hypertriglyceridemia in Type 2 Diabetes Mellitus. Int J
Endocrinol Metab. 2017;15(2):805

37 Bowie A, Owens D, Collins P, et al. Glycosylated low density lipoprotein is more sensitive to oxidation: implications for the diabetic patient? Atherosclerosis. 1993;102(1):63-67.

38 Domingo-Espín J, Nilsson O, Bernfur K, et al. Site-specific glycations of apolipoprotein A-I lead to differentiated functional effects on lipid-binding and on glucose metabolism. Biochim Biophys Acta. 2018;(18)30184-30194.

39 Zhang LS, Sato H, Yang Q, et al Apolipoprotein A-V is present in bile and its secretion increases with lipid absorption in Sprague-Dawley rats. Am J Physiol Gastrointest Liver Physiol. 2015 ;309(11): 918-925.

40 Graf GA, Li WP, Gerard RD, et al. Coexpression of ATP-binding cassette proteins ABCG5 and ABCG8 permits their transport to the apical surface. $J$ Clin Invest. 2002;110(5):659-69.

41 Repa JJ, Berge KE, Pomajzl C, et al. Regulation of ATP-binding cassette sterol transporters $\mathrm{ABCG} 5$ and $\mathrm{ABCG} 8$ by the liver $\mathrm{X}$ receptors alpha and beta . J Biol Chem. 2002;277(21):18793-800.

42 Benítez-Santana T, Hugo SE, Schlegel. A Role of Intestinal LXRo in Regulating Post-prandial Lipid Excursion and Diet-Induced Hypercholesterolemia and Hepatic Lipid Accumulation. Front Physiol. 2017;8:280.

43 Gregg EW, Chen H, Wagenknecht LE, et al. Look AHEAD Research Group. Association of an intensive lifestyle intervention with remission of type 2 diabetes. JAMA. 2012;308(23):2489-2496

44 Swift DL, Houmard JA, Slentz CA, et al. Effects of aerobic training with and without weight loss on insulin sensitivity and lipids. PLoS One. 2018;13(5):637.

45 Lean ME, Leslie WS, Barnes AC, et al. Primary care-led weigh management for remission of type 2 diabetes (DiRECT): an open-label, cluster-randomised trial. Lancet. 2018;391(10120):541-551.

46 Wing RR, Marcus MD, Salata R, et al. Effects of a very-low-calorie diet on long-term glycemic control in obese type 2 diabetic subjects. Arch Intern Med. 1991;151(7):1334-1340

47 Perry RJ, Peng L, Cline GW, et al. Mechanisms by which a Very-LowCalorie Diet Reverses Hyperglycemia in a Rat Model of Type 2 Diabetes. Cell Metab. 2018;27(1):210-221

48 Lim EL, Hollingsworth KG, Aribisala BS, et al. Reversal of type 2 diabetes: normalisation of beta cell function in association with decreased pancreas and liver triacylglycerol. Diabetologia. 2011;54(10):2506-2514.

49 Boyer M, Mitchell PL, Poirier DP, et al. Impact of a 1-year lifestyle modification program on cholesterol efflux capacities in men with abdominal obesity and dyslipidemia. Am J Physiol Endocrinol Metab. 2018.

50 Lin HP, Baghdasarian S, Singer MR, et al. Dietary Cholesterol, Lipid Levels, and Cardiovascular Risk among Adults with Diabetes or Impaired Fasting Glucose in the Framingham Offspring Study. Nutrients. 2018;10(6):770.

51 Soliman GA. Dietary Cholesterol and the Lack of Evidence in Cardiovascular Disease. Nutrients. 2018;10(6):780.

52 Adams TD, Davidson LE, Litwin SE, et al. Weight and Metabolic Outcomes 12 Years after Gastric Bypass. $N$ Engl $J$ Med. 2017;377:1143-1155.

53 Clifton PM, Keogh JB. Effects of Different Weight Loss Approaches on CVD Risk. Current atherosclerosis rep. 2018.

54 Cummings DE, Rubino F. Metabolic surgery for the treatment of type 2 diabetes in obese individuals. Diabetologia. 2018;61(2):257-264. 
55 Jakobsen GS, Småstuen MC, Sandbu R, et al. Association of Bariatric Surgery vs Medical Obesity Treatment With Long-term Medical Complications and Obesity-Related Comorbidities. JAMA. 2018;319(3):291-301.

56 Ikramuddin S, Korner J, Lee WJ, et al. Lifestyle Intervention and Medical Management With vs Without Roux-en-Y Gastric Bypass and Control of Hemoglobin A1c, LDL Cholesterol, and Systolic Blood Pressure at 5 Years in the Diabetes Surgery Study. JAMA. 2018;319(3):266-278.

57 le Roux CW, Heneghan HM. Bariatric Surgery for Obesity. Med Clin North Am. 2018;102(1):165-182.

$58 \mathrm{Xu} \mathrm{L}$, Borges MC, Hemani G, et al. The role of glycaemic and lipid risk factors in mediating the effect of BMI on coronary heart disease: a two-step, two-sample Mendelian randomisation study. Diabetologia. 2017;60(11):2210-2220

59 Lawler PR, Akinkuolie AO, Harada P, et al. JUPITER Trial Residua Risk of Atherosclerotic Cardiovascular Events in Relation to Reductions in Very-Low-Density Lipoproteins. J Am Heart Assoc. 2017;6(12):7402.

60 Nägga K, Gustavsson AM, Stomrud E, et al. Increased midlife triglycerides predict brain $\beta$-amyloid and tau pathology 20 years later. Neurology. 2018;90(1):73-81.

61 Croyal M, Kaabia Z, León L, et al Fenofibrate decreases plasma ceramide in type 2 diabetes patients: A novel marker of CVD?. Diabetes Metab. 2018;44(2):143-149

62 Vallejo-Vaz AJ, Packard CJ, Ray KK. Response by Vallejo-Vaz et al to Letters Regarding Article, "Low-Density Lipoprotein Cholestero Lowering for the Primary Prevention of Cardiovascular Disease Among Men With Primary Elevations of Low-Density Lipoprotein Cholesterol Levels of $190 \mathrm{mg} / \mathrm{dL}$ or Above: Analyses From the WOSCOPS (West of Scotland Coronary Prevention Study) 5-Year Randomized Trial and 20-Year Observational Follow-Up". Circulation. 2018 29;137(22):2419-2420.

63 Nordestgaard BG. Triglyceride-Rich Lipoproteins and Atherosclerotic Cardiovascular Disease: New Insights From Epidemiology, Genetics, and Biology. Circ Res. 2016 ;118(4):547-63

64 Dron JS, Hegele RA. Polygenic influences on dyslipidemias. Curr Opin Lipidol. $2018 ; 29(2): 133-143$

65 Nakano T, Nakajima K, Niimi M, et al Detection of apolipoproteins B-48 and B-100 carrying particles in lipoprotein fractions extracted from human aortic atherosclerotic plaques in sudden cardiac death cases. Clin Chim Acta. 2008;390(1-2):38-43.

66 Masuda D, Sugimoto T, Tsujii K, et al Correlation of fasting serum apolipoprotein B-48 with coronary artery disease prevalence. Eur J Clin Invest. 2012;42(9):992-999.

67 Shah AS, Tan L, Long JL, et al. Proteomic diversity of high density lipoproteins: our emerging understanding of its importance in lipid transport and beyond. J Lipid Res. 2013;54(10):2575-2585

68 Nofer, JR, Brodde MF, Kehrel BE. High-density lipoproteins, platelets and the pathogenesis of atherosclerosis. Clin Exp Pharmacol Physiol. 2010;37:726-735.

69 Muller C, Salvayre R, Negre-Salvayre A, et al. HDLs inhibit endoplasmic reticulum stress and autophagic response induced by oxidized LDLs. Cell Death Differ. 2011;18(5):817-828.

70 Fryirs, MA, Barter PJ, Appavoo M, et al. Effects of high-density lipoproteins on pancreatic beta-cell insulin secretion. Arterioscler Thromb Vasc Biol. 2010;30:1642-1648.

71 Lake NJ, Taylor RL, Trahair H, et al. TRAK2, a novel regulator of ABCA1 expression, cholesterol efflux and HDL biogenesis. Eur Heart J. 2017;38(48):3579-3587.
72 Sacks FM, Jensen MK. From High-Density Lipoprotein Cholesterol to Measurements of Function: Prospects for the Development of Tests for High-Density Lipoprotein Functionality in Cardiovascular Disease. Arterioscler Thromb Vasc Biol. 2018 Mar;38(3):487-499

73 Trigueros-Motos L, van Capelleveen JC, Torta F, et al ABCA8 Regulates Cholesterol Efflux and High-Density Lipoprotein Cholesterol Levels. Arterioscler Thromb Vasc Biol. 2017;37(11):2147-2155.

74 Waldman B, Jenkins AJ, Davis TM, et al. FIELD Study Investigators. HDL-C and HDL-C/ApoA-I predict long-term progression of glycemia in established type 2 diabetes. Diabetes Care. 2014 ;37(8):2351-2358.

75 Mc Queen MJ, Hawken S, Wang X, et al. INTERHEART study investigators. Lipids, lipoproteins, and apolipoproteins as risk markers of myocardial infarction in 52 countries (the INTERHEART study): a case-control study. Lancet. 2008;372(9634):224-233.

76 Borja MS, Hammerson B, Tang C, et al. The European Prospective Investigation Into Cancer-Norfolk Prospective Population Study, the Atherosclerosis Risk in Communities Study, Apolipoprotein A-I exchange is impaired in metabolic syndrome patients asymptomatic for diabetes and cardiovascular disease PLoS One. J Am Heart Assoc. 2017;12(8):217.

77 Melchior JT, Street SE, Andraski AB, et al. Apolipoprotein A-II alters the proteome of human lipoproteins and enhances cholesterol efflux from ABCA1. J Lipid Res. 2017;58(7):1374-1385.

78 Feng X, Gao X, Yao Z, et al. Low apoA-I is associated with insulin resistance in patients with impaired glucose tolerance: a cross-sectional study. Lipids Health Dis. 2017;16(1):69.

79 Rütti S, Ehses JA, Sibler RA, et al. Low- and high-density lipoproteins modulate function, apoptosis, and proliferation of primary human and murine pancreatic beta-cells. Endocrinology. 2009;150(10):4521-4530

80 Pourmousa M, Song HD, He Y, et al. Tertiary structure of apolipoprotein A-I in nascent high-density lipoproteins. Proc Natl Acad Sci USA. 2018;115(20):5163-5168.

81 Ritsch A, Scharnagl H, Marz, W. HDL cholesterol efflux capacity and cardiovascular events. N Engl J Med. 2015;372:1870-1871.

82 Talbot CPJ, Plat J, Joris PJ, et al HDL cholesterol efflux capacity and cholesteryl ester transfer are associated with body mass, but are not changed. Atherosclerosis. 2018;274:23-28.

83 Lincoff AM, Nicholls SJ, Riesmeyer JS, et al. ACCELERATE Investigators. Evacetrapib and Cardiovascular Outcomes in High-Risk Vascular Disease. N Engl J Med. 2017;376(20):1933-1942.

84 Bowman L, Hopewell JC, Chen F, et al. Effects of Anacetrapib in Patients with Atherosclerotic Vascular Disease. $N$ Engl $\mathrm{J}$ Med. 2017;377(13):1217-1227.

85 Di Angelantonio E, Sarwar N, Perry P, et al. Major lipids, apolipoproteins, and risk of vascular disease. JAMA. 2009;302:1993-2000.

86 Barter PJ, Rye K-A. The rationale for using apoA-I as a clinical marker of cardiovascular risk. J Intern Med. 2006;259:447-454.

87 Mineo C, Shaul PW. Novel biological functions of high-density lipoprotein cholesterol. Circ Res. 2012;111:1079-1090.

88 van Capelleveen JC, Bochem AE, Boekholdt SM, et al. Association of High-Density Lipoprotein-Cholesterol Versus Apolipoprotein A-I With Risk of Coronary Heart Disease: The European Prospective Investigation Into Cancer-Norfolk Prospective Population Study, the Atherosclerosis Risk in Communities Study, and the Women's Health Study. J Am Heart Assoc. 2017;6(8).

89 Sun Y, Lee J, Ma RC, et al. Serum high-density lipoprotein cholesterol is a protective predictor of executive function in older patients with diabetes mellitus. J Diabetes Investig. 2018. 
90 Domingo-Espín J, Nilsson O, Bernfur K, et al. Site-specific glycations of apolipoprotein A-I lead to differentiated functional effects on lipidbinding and on glucose metabolism. Biochim Biophys Acta. 2018.

91 Frame NM, Jayaraman S, Gantz DL, et al. Serum amyloid A selfassembles with phospholipids to form stable protein-rich nanoparticles with a distinct structure: A hypothetical function of SAA as a "molecular mop" in immune response. J Struct Biol. 2017; 200(3):293-302.

92 Gomez D, Owens GK. Smooth muscle cell phenotypic switching in atherosclerosis. Cardiovasc Res. 2012;95(2):156-164.

93 Zhang X, Chen J, Wang S. Serum Amyloid A Induces a Vascular Smooth Muscle Cell Phenotype Switch through the p38 MAPK Signaling Pathway. Biomed Res Int. 2017;2017:379.

94 Griffiths K, Pazderska A, Ahmed M, et al .Type 2 Diabetes in Young Females Results in Increased Serum Amyloid A and Changes to Features of High Density Lipoproteins in Both HDL2 and HDL3.J Diabetes Res. 2017.

95 Luo M, Liu A, Wang S, et al. ApoCIII enrichment in HDL impairs HDLmediated cholesterol efflux capacity. Sci Rep. 2017 ;24;7(1):2312

96 Aroner SA, Yang M, Li J, et al. Apolipoprotein C-III and High-Density Lipoprotein Subspecies Defined by Apolipoprotein C-III in Relation to Diabetes Risk. Am J Epidemiol. 2017 15;186(6):736-744

97 Santulli G. Angiopoietin-like proteins: a comprehensive look. Front Endocrinol (Lausanne). 2014;5:4.

98 Zhu P, Goh YY, Chin HF, et al. Angiopoietin-like 4: a decade of research. Biosci Rep. 2012 ;32(3):211-219.

99 Mehta N, Qamar A, Qu L, et al. Differential association of plasma angiopoietin-like proteins 3 and 4 with lipid and metabolic traits. Arterioscler Thromb Vasc Biol. 2014;34:1057-1063.

100 Smart-Halajko MC, Robciuc MR, Cooper JA, et al. The relationship between plasma angiopoietin-like protein 4 levels, angiopoietin-like protein 4 genotype, and coronary heart disease risk. Arterioscler Thromb Vasc Biol. 2010;30(11):2277-2282.

101 Tjeerdema N, Georgiadi A, Jonker JT, et al. Inflammation increases plasma angiopoietin-like protein 4 in patients with the metabolic syndrome and type 2 diabetes. BMJ Open Diabetes Res Care. 2014;2:34

102 Yang LY, Yu CG, Wang XH, et al. Angiopoietin-Like Protein 4 is a High-Density Lipoprotein (HDL) Component for HDL Metabolism and Function in Nondiabetic Participants and Type-2 Diabetic Patients. J Am Heart Assoc. 2017;6(6).

103 Yang LY, Wang XH, Yuan SS, et al. Different relationship between ANGPTL3 and HDL components in female non-diabetic subjects and type-2 diabetic patients. Cardiovasc Diabetol. 2016;15(1):132.

104 Mandard S, Zandbergen F, van Straten E, et al. The fasting-induced adipose factor/angiopoietin-like protein 4 is physically associated with lipoproteins and governs plasma lipid levels and adiposity. J Biol Chem. 2006;281:934-944.
105 Helgadottir A, Gretarsdottir S, Thorleifsson G, et al. Variants with large effects on blood lipids and the role of cholesterol and triglycerides in coronary disease. Nat Genet. 2016;48(6):634-639.

106 Ando Y, Shimizugawa T, Takeshita S, et al. Decreased expression of angiopoietin-like 3 is protective against atherosclerosis in apoE-deficient mice. J Lipid Res. 2003 ;44(6):1216-1223.

107 Dewey FE, Gusarova V, Dunbar RL, et al. Genetic and Pharmacologic Inactivation of ANGPTL3 and Cardiovascular Disease. $N$ Engl J Med. 2017;377(3):211-221

108 Graham MJ, Lee RG, Brandt TA, et al. Cardiovascular and Metabolic Effects of ANGPTL3 Antisense Oligonucleotides. $N$ Engl $\mathrm{J} \mathrm{Med}$. 2017;377(3):222-232

109 Tall AR. Increasing Lipolysis and Reducing Atherosclerosis. $N$ Engl $J$ Med. 2017;377(3):280-283

110 Shimamura M, Matsuda M, Yasumo H, et al. Angiopoietin-like protein3 regulates plasma HDL cholesterol through suppression of endothelial lipase. Arterioscler Thromb Vasc Biol. 2007;27(2):366-372.

111 de Souza JA, Vindis C, Hansel B, et al. Metabolic syndrome features small, apolipoprotein A-I-poor, triglyceride-rich HDL3 particles with defective anti-apoptotic activity. Atherosclerosis. 2008;197(1):84-94.

112 Hansel B, Bonnefont-Rousselot D, Orsoni A, et al. Lifestyle intervention enhances high-density lipoprotein function among patients with metabolic syndrome only at normal low-density lipoprotein cholesterol plasma levels. J Clin Lipidol. 2016;10(5):1172-1181.

113 Lee JS, Chang PY, Zhang Y, et al. Triglyceride and HDL-C Dyslipidemia and Risks of Coronary Heart Disease and Ischemic Stroke by Glycemic Dysregulation Status: The Strong Heart Study. Diabetes Care. 2017;40(4):529-537.

114 Russo GT, De Cosmo S, Viazzi F, et al. AMD-Annals Study Group. Plasma Triglycerides and HDL-C Levels Predict the Development of Diabetic Kidney Disease in Subjects With Type 2 Diabetes: The AMD Annals Initiative. Diabetes Care. 2016;39(12):2278-2287

115 Shiu SW, Wong Y, Tan KC. Pre- $\beta 1$ HDL in type 2 diabetes mellitus. Atherosclerosis. 2017;263:24-28.

116 Voight BF, Peloso GM, Orho-Melander M, et al, Plasma HDL cholesterol and risk of myocardial infarction: a mendelian randomisation study. Lancet. 2012;380(9841):572-580.

117 Dron JS, Hegele RAGenetics of Triglycerides and the Risk of Atherosclerosis. Curr Atheroscler Rep. 2017;19(7):31.

118 Russo GT, De Cosmo S, Viazzi F, et al. AMD-Annals Study Group. Triglyceride and HDL-C Dyslipidemia and Risks of Coronary Heart Disease and Ischemic Stroke by Glycemic Dysregulation Status: The Strong Heart Study. Diabetes Care. 2017;40:529-537

119 Benítez-Santana T, Hugo SE, Schlegel A. Role of Intestinal LXRo in Regulating Post-prandial Lipid Excursion and Diet-Induced Hypercholesterolemia and Hepatic Lipid Accumulation. Front Physiol. 2017;9;8:280. 